\title{
Thiel embalming in neonates: methodology and benefits in medical training
}

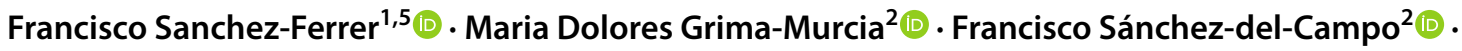 \\ Maria Luisa Sánchez-Ferrer ${ }^{3}$ (iD $\cdot$ Eduardo Fernández-Jover ${ }^{4}$ [C]
}

Received: 28 December 2021 / Accepted: 18 January 2022 / Published online: 8 February 2022

(c) The Author(s) 2022

\begin{abstract}
Current teaching and training methods for surgical techniques in the pediatric population involve artificial models (manikins), animals or adult human cadavers embalmed using various techniques. We found no references in the literature concerning the use of the Thiel method in the pediatric population. The aim of this study, therefore, was to assess the viability of using pediatric human cadavers embalmed through Thiel's technique and to compare them with standard pediatric manikins. After donation of a 24-week stillborn, the Thiel technique was carried out for fixation following the usual protocol. A video recording with eye-tracking glasses was used to perform an examination, and techniques. The same procedures were conducted on a pediatric manikin. Medical students, medical residents and physicians were asked to respond to questions in an online survey after being shown the video. A total of 92 responses were obtained. The Thiel-embalmed stillborn was assessed as superior to the manikin in all items. Our study confirmed that this technique is feasible even with extremely small donors. The value of this form of preservation for medical training is not widely known though it is receiving increasing interest. Our results show that Thiel fixation in pediatrics is clearly more highly valued than a manikin and offers great potential. This innovative application of the Thiel method in the pediatric population is technically possible. It poses no additional difficulties and is very positively assessed for undergraduate and postgraduate teaching.
\end{abstract}

Keywords Donation $\cdot$ Thiel embalming $\cdot$ Cadavers $\cdot$ Pediatrics $\cdot$ Medical training

\section{Introduction}

Human cadavers are not only used for the study of human anatomy. They also play an important role as anatomical

Francisco Sánchez Ferrer and Grima Murcia contributed equally to this work.

\section{Francisco Sanchez-Ferrer}

f.sanchez@umh.es

1 Medical School, University of Miguel Hernández de Elche, Sant Joan d'Alacant, Spain

2 Anatomical Innovation Service, University of Miguel Hernández de Elche, Sant Joan d'Alacant, Spain

3 Medical School, University of Murcia, Murcia, Spain

4 Bioengineering Institute, University of Miguel Hernández de Elche, CIBER BBN, Elche, Spain

5 Pediatric Service, Pediatric Cardiology Unit, San Juan de Alicante University Hospital (Alicante, Spain), Carretera Nacional 332, Alicante-Valencia s/n, 03550 Sant Joan d'Alacant, Spain models in situations where it is impractical, illegal, or unethical to work with patients, as is the case with surgical specialty training or intensive care unit techniques (Jaung et al. 2011; Groscurth et al. 2001). The use of cadavers is also helpful in the preclinical development of instruments and procedures, enabling products to be tested safely without risk to patients but with a high degree of similarity.

Although other models such as augmented or virtual reality (Kugelmann et al. 2018), 3D printed models, animals or manikins are also used, it is difficult to achieve the degree of realism offered by the human body. The bodies donated for scientific and training purposes, generously bequeathed by donors, are known as "silent teachers" (Eisma and Wilkinson 2014).

The history of techniques to preserve the human body goes back many centuries and includes natural means such as mummification or freezing and artificial methods such as immersion or arterial injection (Brenner 2014). Embalmed cadavers are employed in education, training, and research with minimal risk of infection, and they allow for prolonged use. Nonetheless, depending on the embalming method, changes in color, mobility, or tissue texture occur (Brenner 
2014). Fresh-frozen bodies are more realistic and flexible, but the time available before deterioration is short and the risk of infection for the user increases. For many years, the most widespread technique worldwide for embalming has involved the use of formalin. Formalin-embalmed cadavers have great durability but are not very useful for surgical training, such as laparoscopy.

An embalming method named after its discoverer, Thiel, has been in use since the 1990s (Thiel 1992b, 2002). Through this form of preservation, the cadavers remain flexible, with coloring, mobility and texture very similar to a living patient. This method also enables preservation (including the internal organs) over a long period of time and is ideal for many activities including examination techniques (Feigl et al. 2020; Aurore et al. 2020), radiological tests and surgery (Odobescu et al. 2014; Benkhadra et al. 2009; Giger et al. 2008). This type of embalming is still relatively unknown and is used in very few places in Europe, possibly because the original text was published in German (Benkhadra et al. 2011).

With respect to training in pediatric patients, both the degree and the specialization are based on references in books, virtual reality, pediatric simulation manikins or clinical practice itself when possible (Harada et al. 2015; Stone et al. 2014; Trudeau et al. 2018; Lopreiato and Sawyer 2015). Indeed, much of this training must be performed on adult human references such as cadavers embalmed using different techniques and found in the anatomy laboratories (Prigge et al. 2014; Wagner et al. 2018).

This study examines the potential use of Thiel embalming for pediatric cadavers donated to science. We evaluate its usefulness and the opinions of students and professionals regarding the results.

\section{Materials and methods}

\section{Technique and procedures}

Embalming was performed using Thiel's technique on a fetus deceased in utero at 24 weeks of gestation, weighing $680 \mathrm{~g}$, donated to the Department of Anatomy and Histology of Miguel Hernández University in 2015. The donation was carried out according to the legislation in force at the time.

Once the cadaver was received in the department, the infusion was performed by cannulating the umbilical vessels with the different fluids used in Thiel's technique, which include monopropylene glycol, ammonium nitrate, potassium nitrate, sodium sulfite, boric acid, chlorocresol, formaldehyde, alcohol and morphine (Thiel 1992a, b, 2002; Eisma and Wilkinson 2014). Subsequently, the cadaver was preserved in embalming fluid for 6 months.

\section{Video recording and comparison between Thiel cadaver and neonatal manikin}

A pediatrician evaluated the different techniques for examination, mobility, ultrasound, lumbar puncture and intubation as well as the general characteristics of the neonate preserved using Thiel's method. The same procedures were carried out on a neonatal manikin.

All these actions were video recorded (using both the Thiel-fixed neonatal cadaver and the manikin) through eyetracking glasses (Tobii Glasses 2), providing a first-person perspective. Throughout the video, a circle can be seen, which indicates where the physician's gaze is focused at all times (see Video).

The TitanR (SonoSite) ultrasound machine with a high frequency linear probe was used for the ultrasound assessment.

\section{Survey for students and professionals}

A voluntary, anonymous online questionnaire was administered for evaluation of the video by medical students, medical residents and attending physicians (Annex 1).

The participants first answered questions regarding demographic information then questions about their comparison of the degree of realism of the Thiel-cadaver and the manikin as well as their assessment of teaching opportunities and which techniques provided the greatest potential. A scale of 1-5 (1 very poor-5 very good) was used for the evaluation of both training options.

\section{Results}

The techniques and procedures for preserving neonatal cadavers are similar to those used for adult cadavers, except that it is more difficult to cannulate the femoral or jugular vein in the neonate, while the umbilical vein is more accessible. We, therefore, cannulated the umbilical vein with a neonatal catheter. The solution used was the same as that routinely used for infusion in adult cadavers.

The results in terms of mobility and texture were comparable to those of an adult cadaver, although in our case the skin color was patchy. The internal organs visualized by ultrasound allowed us to see their basic anatomy. The visualization of the glottis for intubation allowed us to observe the perfect conservation of the oropharynx and glottis.

\section{Survey}

The questionnaire was administered between July and September 2019 and 92 people responded, of whom $77.2 \%$ were women and $22.8 \%$ were men. There was an even distribution across the three target groups with $29.3 \%$ students, $37 \%$ medical residents and $33.7 \%$ attending physicians. 
Responses concerning whether respondents are interested in Thiel courses, how the Thiel method is of interest in laparoscopy and whether they believe that postgraduate teaching is of interest are shown in Fig. 1.

After viewing the video, the responses concerning the Thiel-fixed neonatal cadaver (SST) were significantly higher than for the manikin (Table 1).

Figure 2 depicts the comparison between techniques. In no case was the manikin considered superior to the SST, with the differences being much greater for ultrasound and lumbar puncture.

The scores on the scale from 1 to 5 showed a mean value for the SST of 4.37 and for the manikin of $3.43(p<0.001)$. The comments provided at the end of the survey revealed considerable interest in Thiel's fixation technique and, in many cases, surprise at the lack of knowledge about it.

\section{Discussion}

The use of Thiel embalming is growing due to the applications and opportunities it provides (Boaz et al. 2013, no date). An increasing number of healthcare professionals are using adult cadavers fixed with this method for the study of anatomy, learning techniques, professional training and for testing materials in preclinical research as these activities can present risks in real patients (Boaz and Anderhuber 2009, no date). Thiel-embalmed donor animals are also increasingly used for veterinary training (Nam et al. 2020) as well as in medical training when human donors are scarce.

Since 2016, the occupational exposure limit to formaldehyde was lowered by the European Committee on Hazardous Substances, as formaldehyde had been classified as a human 1B carcinogen. For this reason, in 2018 the Anatomische Gesellschaft working group issued recommendations to limit exposure to formaldehyde in anatomy laboratories (Waschke et al. 2019). The Thiel method on the other hand has no
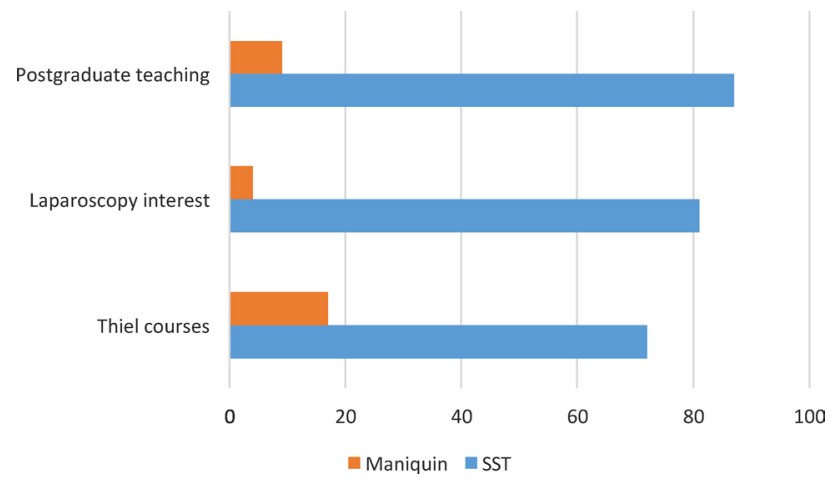

Fig. 1 The percentage of positive or negative responses to interest in Thiel courses, interest in the Thiel method for laparoscopy or general interest for postgraduate teaching
Table 1 Comparison between Thiel-fixed cadaver (SST) and neonatal manikin

\begin{tabular}{llr}
\hline$N=92(\%)$ & Thiel (SST) & Manikin \\
\hline Lifelike & $84(91 \%)$ & $7(7.6 \%)$ \\
Teaching opportunities & $66(71.7 \%)$ & $23(25 \%)$ \\
\hline
\end{tabular}

proven harmful effects. It is also safe at the microbiological level (Rowe et al. 2020).

Various specialties use Thiel-embalmed cadavers for training and learning purposes (Esposito et al. 2015; Hölzle et al. 2012; Wolff et al. 2008; Hassan et al. 2015; Peuker et al. 2001), particularly in laparoscopy (Giger et al. 2008) (at times combined with the use of ultrasound) (Veys et al. 2020), microsurgery (Odobescu et al. 2019), plastic surgery and neurosurgery in combination with nuclear magnetic resonance (Eljamel et al. 2014). These cadavers are also valuable in the management of the airway where the superiority of Thiel-embalmed cadavers over manikins has been demonstrated (Szucs et al. 2016).

Pediatric patients have a different anatomy and characteristics than adults. For this reason, pediatric models are especially important (Ade-Ajayi et al. 2013). A child is not a "small adult" and the differences are greater at a younger age and size. Techniques such as laparoscopy (Ade-Ajayi et al. 2013), placement of a central catheter (Thomas et al. 2013) or neonatal and pediatric intubation (O'Shea et al. 2020; Ernst et al. 2014) are all performed in pediatric training. Many of these techniques are learned with simulators, high-fidelity pediatric manikins (Azzie et al. 2011) or 3D models (Rose et al. 2015) and, in certain cases, on real patients.

Our study presents a smaller human cadaver embalmed using Thiel's method, as described in the references. We were able to verify that this technique is feasible and that the results are similar to those of adult cadavers fixed with this technique (Eisma and Wilkinson 2014), hence the name Small Silent Teacher. The only difference in the fixation is that we obtain access for the infusion through the umbilical vessels due to the greater ease.

Thiel cadavers and our neonatal cadaver in particular present characteristics of color, texture, flexibility, mobility and internal

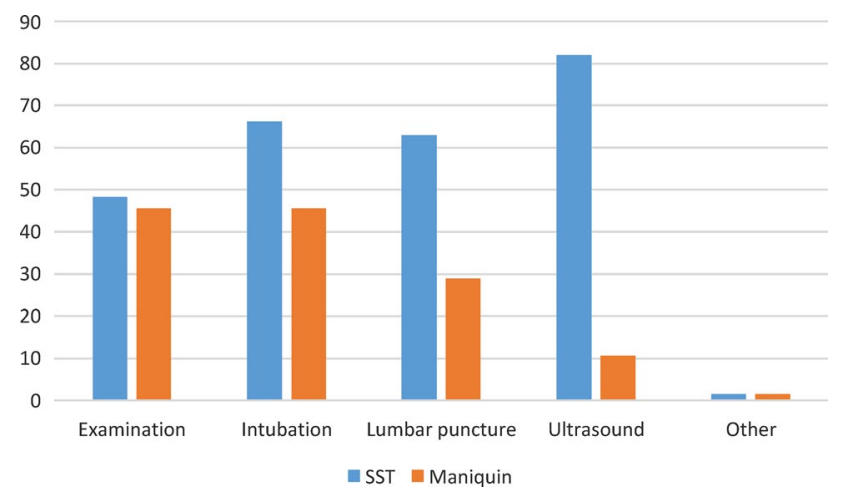

Fig. 2 Survey responses on the usefulness of the Thiel cadaver (SST) and the manikin in different procedures 
organ preservation very similar to the living body. This has been proven in other Thiel-fixed cadavers through radiological tests such as nuclear magnetic resonance, plain-film radiology or ultrasound (Joy et al. 2015; Schramek et al. 2013). The lifelike properties of the specimens are highlighted in the publications involving the Thielembalming technique (Hohmann et al. 2019; Hammer et al. 2015).

Medical students and professionals assessed the neonatal Thiel-fixed cadaver very favorably and found it to be superior to the pediatric manikin with which it was compared. Those surveyed highlighted the realistic quality, the teaching opportunities it offers and its particular usefulness for techniques such as ultrasound, lumbar puncture or intubation. A single cadaver can be used for multiple consecutive procedures, even over several years, which makes its use efficient. Also, unlike formaldehyde, Thiel embalming is a safer form of preservation (Waschke et al. 2019).

We can affirm that Thiel's embalming method is technically possible and uncomplicated in pediatric patients, offering teaching and research opportunities similar to those offered by adult Thiel cadavers. The limitations we observed are the reduced number of donated bodies in the pediatric population, firstly because of the low mortality rate and in many cases because of the lack of knowledge about the option of donation, which in many stillbirths would be possible.

\section{Conclusions}

The novel use of Thiel's embalming technique in neonates provides learning and training opportunities for students and physicians working with pediatric populations. Human anatomy changes with age and this method offers a combination of a high level of fidelity to the living child, safety in its use and preservation of the body.

\section{Annex}

Link to Video: https://www.youtube.com/watch?reload=9\&

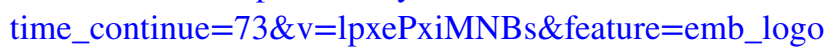

Link to Survey: https://docs.google.com/forms/d/e/ 1FAIpQLScXJgrIvx X_wPa9s 8KPelpSirIFqPL-j 12li H1f5G-5R7keqw/viewform?vc $=0 \& \mathrm{c}=0 \& \mathrm{w}=1$

\section{Anatomical model assessment survey}

You will watch a short video with two types of models that can be used as a teaching aid for instruction in various techniques.

Afterwards, you will be given a short survey, which will not take more than 5 minutes of your time. Thank you very much.

\section{Gender:}

Woman / Man

Age: Years

Professional category: Medical student / Medical resident / Attending physician

If you work, where do you work? Hospital / Primary Care / Other

If you work, is this in a surgical specialty? Yes / No

Do you think that teaching outside the field of clinical care is useful? Yes / No

Viewing the video:

Which of the two types of simulators is most lifelike?

Manikin / Thiel-fixed donated body 
What kind of simulators do you think provide more teaching opportunities?

Manikin / Thiel-fixed donated body

For which technique do you consider that the manikin offers advantages over the Thiel-fixed donated body? (Select all that apply)

Examination / Intubation / Lumbar Puncture / Ultrasound / Other

For which technique do you consider that the Thiel-fixed donated body offers advantages over the manikin? (Select all that apply)

Examination / Intubation / Lumbar Puncture / Ultrasound / Other

Would you like to be offered postgraduate training courses with Thiel-fixed donated bodies for learning various techniques?

Yes / No

On a scale of 1 to 5, rate the manikin as a teaching aid (1 very poor - 5 very good)

On a scale of 1 to 5, rate the Thiel-fixed donor cadaver as a teaching aid (1 very poor - 5 very good)

Courses in laparoscopy/surgery are being held in adult Thiel models. Do you think this would be of interest in pediatrics?

Yes / No

Please leave your comments and suggestions here.

Supplementary Information The online version contains supplementary material available at https://doi.org/10.1007/s12565-022-00650-1.

Acknowledgements The authors thank Maria Repice for her help with the English version of the text. The authors sincerely thank those who have donated their bodies to science so that anatomical research could be performed. Results from such research can potentially increase mankind's overall knowledge that can then improve patient care. Therefore, these donors and their families deserve our highest gratitude.

Author contributions The authors declare their responsibility for the following tasks (according to their initials). Francisco Sánchez-Ferrer (FSF), María Dolores Grima Murcia (MDGM), Francisco Sánchez del Campo (FSC), Maria Luisa Sánchez Ferrer (MLSF) Eduardo Fernández Jover (EFJ). FSF, MDGM, FSC, MLSF, EFJ: conception and design of the study, data collection, analysis and interpretation. FSF, MDGM, FSC, MLSF, EFJ: drafting and critical revision of the manuscript, with important intellectual contributions. FSF, MDGM, MLSF,
FSC, EFJ: approval of the final draft for publication. All authors, having revised and discussed the manuscript, take responsibility and serve as guarantors for the accuracy and integrity of the report.

Funding Open Access funding provided thanks to the CRUE-CSIC agreement with Springer Nature. Not applicable. This project has not received any funding.

\section{Declarations}

Conflict of interest The authors declare that they have no conflicts of interest.

Ethical statement The authors declare that they have followed all the ethical guidelines for publication. 
Open Access This article is licensed under a Creative Commons Attribution 4.0 International License, which permits use, sharing, adaptation, distribution and reproduction in any medium or format, as long as you give appropriate credit to the original author(s) and the source, provide a link to the Creative Commons licence, and indicate if changes were made. The images or other third party material in this article are included in the article's Creative Commons licence, unless indicated otherwise in a credit line to the material. If material is not included in the article's Creative Commons licence and your intended use is not permitted by statutory regulation or exceeds the permitted use, you will need to obtain permission directly from the copyright holder. To view a copy of this licence, visit http://creativecommons.org/licenses/by/4.0/.

\section{References}

Ade-Ajayi N et al (2013) Paediatric laparoscopic hernia repair: ex vivo skills in the reduced training era. Afr J Paediatr Surg 10(2):95. https://doi.org/10.4103/0189-6725.115031

Aurore V et al (2020) Anatomy of the female pelvic nerves: a macroscopic study of the hypogastric plexus and their relations and variations. J Anat 237(3):487-494. https://doi.org/10.1111/joa.13206

Azzie G et al (2011) Development and validation of a pediatric laparoscopic surgery simulator. J Pediatr Surg. https://doi.org/10.1016/j. jpedsurg.2011.02.026

Benkhadra M et al (2009) Comparison of fresh and Thiel's embalmed cadavers according to the suitability for ultrasound-guided regional anesthesia of the cervical region. Surg Radiol Anat 31(7):531-535. https://doi.org/10.1007/s00276-009-0477-z

Benkhadra M et al (2011) Is Thiel's embalming method widely known? A world survey about its use. Surg Radiol Anat. https://doi.org/ 10.1007/s00276-010-0705-6

Boaz NT, Anderhuber F (2009) The uses of soft embalming for cadaver-based dissection, instruction in gross anatomy, and training of physicians. FASEB J 23:480.3. https://doi.org/10. 1096/fasebj.23.1_supplement.480.3

Boaz NT et al (2013) New applications in anatomical education using soft-embalmed cadavers. FASEB J 27:960.34. https://doi. org/10.1096/FASEBJ.27.1_SUPPLEMENT.960.34

Brenner E (2014) Human body preservation-old and new techniques. J Anat 224(3):316-344. https://doi.org/10.1111/joa. 12160

Eisma R, Wilkinson T (2014) From "silent teachers" to models. PLoS Biol 12(10):e1001971. https://doi.org/10.1371/journal. pbio. 1001971

Eljamel S et al (2014) Evaluation of Thiel cadaveric model for MRIguided stereotactic procedures in neurosurgery. Surg Neurol Int 5(9):404. https://doi.org/10.4103/2152-7806.140199

Ernst KD et al (2014) Weekly and consecutive day neonatal intubation training: comparable on a pediatrics clerkship. Acad Med 89(3):505-510. https://doi.org/10.1097/ACM.0000000000 000150

Esposito C et al (2015) European society of pediatric endoscopic surgeons (ESPES) guidelines for training program in pediatric minimally invasive surgery. Pediatr Surg Int 31(4):367-373. https:// doi.org/10.1007/s00383-015-3672-5

Feigl G et al (2020) The intercarotid or alar fascia, other cervical fascias, and their adjacent spaces - a plea for clarification of cervical fascia and spaces terminology. J Anat 237(1):197-207. https://doi. org/10.1111/joa.13175

Giger U et al (2008) Laparoscopic training on Thiel human cadavers: a model to teach advanced laparoscopic procedures. Surg Endosc 22(4):901-906. https://doi.org/10.1007/s00464-007-9502-7
Groscurth P et al (2001) Gross anatomy in the surgical curriculum in Switzerland: improved cadaver preservation, anatomical models, and course development. Anat Rec 265(6):254-256

Hammer $\mathrm{N}$ et al (2015) Comparison of modified thiel embalming and ethanol-glycerin fixation in an anatomy environment: potentials and limitations of two complementary techniques. Anat Sci Educ 8(1):74-85. https://doi.org/10.1002/ase. 1450

Harada K et al (2015) Quantitative pediatric surgical skill assessment using a rapid-prototyped chest model. Minim Invas Ther Allied Technol 24(4):226-232. https://doi.org/10.3109/13645706.2014. 996161

Hassan S et al (2015) Surgical simulation flexor tendon repair using Thiel cadavers: a comparison with formalin embalmed cadavers and porcine models. J Hand Surg (Eur Vol) 40(3):246-249. https://doi.org/10.1177/1753193413520281

Hohmann E et al (2019) The mechanical properties of fresh versus fresh/frozen and preserved (Thiel and Formalin) long head of biceps tendons: a cadaveric investigation. Ann Anat 221:186-191. https://doi.org/10.1016/j.aanat.2018.05.002

Hölzle F et al (2012) Thiel embalming technique: a valuable method for teaching oral surgery and implantology. Clin Implant Dent Relat Res 14(1):121-126. https://doi.org/10.1111/j.1708-8208. 2009.00230.x

Jaung R, Cook P, Blyth P (2011) A comparison of embalming fluids for use in surgical workshops. Clin Anat 24(2):155-161. https:// doi.org/10.1002/ca.21118

Joy J et al (2015) Quantitative assessment of Thiel soft-embalmed human cadavers using shear wave elastography. Ann Anat 202:5256. https://doi.org/10.1016/j.aanat.2015.06.007

Kugelmann D et al (2018) An augmented reality magic mirror as additive teaching device for gross anatomy. Ann Anat 215:71-77. https://doi.org/10.1016/j.aanat.2017.09.011

Lopreiato JO, Sawyer T (2015) Simulation-based medical education in pediatrics. Acad Pediatr. https://doi.org/10.1016/j.acap.2014. 10.010

Nam SM et al (2020) Comparative evaluation of canine cadaver embalming methods for veterinary anatomy education. Anat Sci Int. https://doi.org/10.1007/s12565-020-00547-x

O'Shea JE et al (2020) Neonatal videolaryngoscopy as a teaching aid: the trainees' perspective. Arch Dis Childh Fetal Neonatal Ed. https://doi.org/10.1136/archdischild-2020-319619

Odobescu A et al (2014) A new microsurgical research model using Thiel-embalmed arteries and comparison of two suture techniques. J Plast Reconstr Aesth Surg 67(3):389-395. https://doi. org/10.1016/j.bjps.2013.12.026

Odobescu A et al (2019) High fidelity microsurgical simulation: the thiel model and evaluation instrument. Plast Surg 27(2):118-124. https://doi.org/10.1177/2292550318800324

Peuker ET et al (2001) Surgical procedures in mouth, jaw and facial surgery in Thiel embalmed body donors. Mund-, Kiefer- Und Gesichtschirurgie 5(2):141-143. https://doi.org/10.1007/PL000 10796

Prigge L et al (2014) Clinical anatomy of the maxillary nerve block in pediatric patients. Paediatr Anaesth 24(11):1120-1126. https:// doi.org/10.1111/pan.12480

Rose AS et al (2015) Pre-operative simulation of pediatric mastoid surgery with 3D-printed temporal bone models. Int J Pediatr Otorhinolaryngol 79(5):740-744. https://doi.org/10.1016/j.ijporl. 2015.03.004

Rowe J et al (2020) Microbial growth on soft embalmed cadavers over time. FASEB J 34(S1):1-1. https://doi.org/10.1096/fasebj.2020. 34.s1.02513

Schramek GGR et al (2013) Imaging in anatomy: a comparison of imaging techniques in embalmed human cadavers. BMC Med Educ 13(1):143. https://doi.org/10.1186/1472-6920-13-143 
Stone K et al (2014) Increasing pediatric resident simulated resuscitation performance: a standardized simulation-based curriculum. Resuscitation 85(8):1099-1105. https://doi.org/10.1016/j.resus citation.2014.05.005

Szucs Z et al (2016) Suitability of a preserved human cadaver model for the simulation of facemask ventilation, direct laryngoscopy and tracheal intubation: a laboratory investigation. $\mathrm{Br} \mathrm{J}$ Anaesth 116(3):417-422. https://doi.org/10.1093/bja/aev546

Thiel W (1992a) An arterial substance for subsequent injection during the preservation of the whole corpse. Ann Anat 174(3):197-200

Thiel W (1992b) The preservation of the whole corpse with natural color. Ann Anat 174(3):185-195

Thiel W (2002) Supplement to the conservation of an entire cadaver according to W. Thiel. Ann Anat 184(3):267-269

Thomas SM et al (2013) Simulation training for pediatric residents on central venous catheter placement. Pediatr Crit Care Med 14(9):e416-e423. https://doi.org/10.1097/PCC.0b013e3182 9f5eda

Trudeau MON et al (2018) Comparison of adult and pediatric surgeons: insight into simulation-based tools that may improve expertise among experts. J Laparoendosc Adv Surg Tech 28(5):599-605. https://doi.org/10.1089/lap.2017.0214

Veys R et al (2020) Thiel-embalmed cadavers as a novel training model for ultrasound-guided supine endoscopic combined intrarenal surgery. BJU Int 125(4):579-585. https://doi.org/10.1111/bju.14954

Wagner $\mathrm{M}$ et al (2018) Implementation and evaluation of training for ultrasound-guided vascular access to small vessels using a lowcost cadaver model. Pediatr Crit Care Med 19(11):E611-E617. https://doi.org/10.1097/PCC.0000000000001721

Waschke J et al (2019) Recommendations of the working group of the Anatomische Gesellschaft on reduction of formaldehyde exposure in anatomical curricula and institutes. Ann Anat 221:179-185. https://doi.org/10.1016/j.aanat.2018.10.007

Wolff K-D et al (2008) Thiel embalming technique: a valuable method for microvascular exercise and teaching of flap raising. Microsurgery 28(4):273-278. https://doi.org/10.1002/micr.20484

Publisher's Note Springer Nature remains neutral with regard to jurisdictional claims in published maps and institutional affiliations. 